Rev. Int. Contam. Ambie. 32 (4) 413-424, 2016

DOI: 10.20937/RICA.2016.32.04.05

\title{
OBTENCIÓN DE UN MEDIO ENRIQUECIDO PARA HACER MÁS EFICIENTE LA BIODISPONIBILIDAD DE LOS HIDROCARBUROS INTEMPERIZADOS EN UN SUELO COSTERO
}

\author{
Verónica JIMÉNEZ HERNÁNDEZ* y Ricardo GUERRA SÁNCHEZ
}

Centro de Innovación Aplicada en Tecnologías Competitivas, Asociación Civil. Omega 201, Industrial Delta, León, Guanajuato, México, C.P. 37545.

*Autor para correspondencia: veronicjh@gmail.com

(Recibido junio 2015; aceptado febrero 2016)

Palabras clave: petróleo crudo, intemperismo, recalcitrancia, bioestimulación

\section{RESUMEN}

Los hidrocarburos intemperizados presentan una composición formada principalmente por la fracción pesada del petróleo, los cuales son altamente persistentes. La complejidad química del suelo dificulta la biodegradación de los hidrocarburos. El objetivo de este trabajo fue incrementar la biodisponibilidad de hidrocarburos altamente persistentes, obteniendo las mejores condiciones nutrimentales para que los microorganismos nativos en un consorcio adaptado desarrollaran el máximo potencial de degradación en un suelo contaminado con hidrocarburos intemperizados, a través de un proceso de bioestimulación. Se utilizó un diseño de experimentos factorial fraccionado del tipo Plackett-Burman para encontrar la combinación de nutrientes específicos que aporten los requerimientos mínimos necesarios para que las bacterias hidrocarbonoclastas desarrollen sus procesos metabólicos de manera eficiente. La biodegradación de los hidrocarburos fue estimada con base en el contenido de hidrocarburos de fracción pesada (HFP)/ $\mathrm{kg}$ suelo seco, producción de $\mathrm{CO}_{2} / \mathrm{kg}$ suelo seco, así como la alteración de algunas características fisicas y químicas como el $\mathrm{pH}, \mathrm{CE}(\mu \mathrm{S} / \mathrm{cm})$, porcentaje de humedad y porcentaje de materia orgánica. Este trabajo realiza una contribución al estudio de la biodisponibilidad de los hidrocarburos persistentes mediante el control de las condiciones nutrimentales en un proceso de bioestimulación a través de un diseño de experimentos. Por otro lado, la adición de un consorcio microbiano adaptado a concentraciones de $1 \%$ de crudo Maya incrementó la degradación de los hidrocarburos intemperizados en el suelo costero hasta en un $97 \%$, expresados como HFP en un periodo de 28 días de degradación.

Key words: crude oil, weathering, recalcitrance, biostimulation

\begin{abstract}
The weathered hydrocarbons are mainly constituted of highly persistent heavy petroleum fractions. Thus, the soil chemical complexity delays the hydrocarbon biodegradation. The objective of this study was to increase the bioavailability of highly persistent compounds by obtaining the greatest nutritional conditions for indigenous microorganisms. A consortium was previously adapted in order to achieve the full potential of degradation through the biostimulation process which was carried out by the Plackett-Burman
\end{abstract}


analytic method experiments. These experiments conducted to a specific combination of nutrients, which provided the minimum requirements for the hydrocarbonoclastic bacteria to efficiently improve their metabolic processes. The biodegradation of hydrocarbons were estimated based on the heavy oil $/ \mathrm{kg}$ dry soil, $\mathrm{CO}_{2}$ production $/ \mathrm{kg}$ dry soil, as well as the alteration of certain physicochemical characteristics, such as $\mathrm{pH}$, EC $(\mu \mathrm{S} / \mathrm{cm})$, moisture and organic matter percentage. In conclusion, these analyses provide a contribution to the study of bioavailability of weathered hydrocarbons by controlling conditions in a nutrient biostimulation process and through the design of experiments. Likewise, a microbial consortium adapted to a $1 \%$ concentration of Mayan crude, resulted in an up to $97 \%$ increase in hydrocarbon degradation in the coastal weathered soil, which were expressed as a heavy fraction of hydrocarbons in a period of 28 days of degradation.

\section{INTRODUCCIÓN}

Un gran número de estudios de degradación de hidrocarburos en el suelo van dirigidos a promover la desorción y solubilización de hidrocarburos recalcitrantes para hacerlos biodisponibles en la fase libre mediante la aplicación de métodos físicos, químicos, térmicos o biológicos. Sin embargo, en algunos casos se han desarrollado efectos adversos que ocasionan la perturbación del sitio. Por ejemplo, la generación de subproductos aún más tóxicos y carcinogénicos que el contaminante principal (Satyanarayana et al. 2012). En el caso de los métodos biológicos si no existen la condiciones ideales de nutrientes, humedad, textura, $\mathrm{pH}$ y microorganismos, entre otros, se pueden presentar efectos antagónicos entre la microbiota nativa y los microorganismos adaptados (Mihelcic et al. 1993, Antizar et al. 2009 ). En México, el crudo Maya es el más producido respecto a otros tipos, lo que lo convierte en un contaminante potencialmente perjudicial en los ecosistemas costeros puesto que es generalmente en estas zonas donde se encuentran los yacimientos de crudo y a su vez una intensa actividad petrolera. En México la actividad petrolera tiene más de 50 años, por lo que en las últimas décadas el número de sitios sin remediar ha aumentado significativamente. Lo anterior, resulta en una acumulación de hidrocarburos intemperizados, los cuales son difíciles de degradar por los microorganismos nativos incluso en condiciones controladas (Hueseman et al. 1993, Adams et al. 2009). Por lo tanto es necesario llevar a cabo procedimientos que permitan incrementar la biodisponibilidad de los hidrocarburos persistentes de manera más eficiente, a un bajo costo y sin ocasionar daños al ambiente.

Un diseño de experimentos del tipo Plackett Burman factorial fraccionado con 12 experimentos es un diseño de barrido (Montgomery 1991) que puede emplearse para maximizar la biodegradación de los hidrocarburos persistentes en el ambiente mediante el control de parámetros como: humedad, temperatura, aireación, biomasa y nutrientes. La adición óptima de nutrientes es determinante para hacer más eficiente el metabolismo microbiano. Se requiere profundizar en los requerimientos nutrimentales y en la influencia que tienen las condiciones del suelo, la naturaleza de la microbiota y las características del contaminante, que afectan la bioasimilación de los nutrientes.

La biodisponibilidad es la facilidad relativa con la que un compuesto puede ser usado, biotransformado o degradado por los microorganismos presentes en el suelo. Esto se entiende como el efecto neto entre la desorción y la biodegradación (García 2003), es aquí donde la aportación de nutrientes es determinante para establecer un equilibrio entre ambos efectos. La adición optima de nutrientes durante el tratamiento de un suelo contaminado y la aplicación de un consorcio microbiano adaptado a la fracción pesada del crudo Maya promueven la desorción y biotransformación de los hidrocarburos químicamente complejos al desarrollar en los microorganismos la actividad enzimática mediante cofactores específicos. Estos influyen en el rompimiento de las estructuras complejas de los hidrocarburos que la microbiota nativa no puede realizar. En este sentido los microorganismos adaptados son capaces de sintetizar subproductos como ácidos carboxílicos, alcoholes y ácidos grasos, que promueven la solubilización de los contaminantes secuestrados en los poros del suelo y de los nutrientes ligados mediante enlaces químicos a la fracción mineral, los cuales son aprovechados como fuente de energía por los mismos microorganismos.

El monitoreo y manejo de los parámetros de operación en una tecnología de biorremediación implica tiempo y costo. No obstante, es determinante controlar la adición de nutrientes ya que un exceso o una baja concentración de éstos puede inhibir el proceso metabólico de los microorganismos. Los hidrocarburos 
intemperizados son altamente persistentes, por lo que la ventaja de implementar un proceso de bioestimulación es la poca o nula perturbación al suelo, así como el costo-beneficio en comparación a otros métodos de remediación de suelos. El objetivo de este trabajo se enfocó en alcanzar la mayor degradación de los hidrocarburos intemperizados mediante la optimización de un proceso de bioestimulación al adicionar una concentración de nutrientes mínima necesaria para eficientizar el metabolismo de los microorganismos asociados a la degradación de los hidrocarburos.

\section{MATERIALES Y MÉTODOS}

\section{Suelo}

El suelo contaminado con hidrocarburos intemperizados se colectó de una región costera, en el municipio de Agua Dulce, Veracruz, México, que está ubicado en las coordenadas $18^{\circ} 09^{\prime \prime}$ latitud norte y $94^{\circ} 08^{\prime \prime}$ longitud oeste y una altitud de $20 \mathrm{msnm}$. La vegetación cercana al sitio está compuesta por totora (Typha latifolia), cañas (Phragmites australis) y leguminosas (Mimosa spp.). La temperatura promedio es de $25.6{ }^{\circ} \mathrm{C}$ y la precipitación anual de $2041 \mathrm{~mm}$ (INAFED 2010). El sitio se ubica entre dos municipios (Coatzacoalcos y Minatitlán), en los cuales existe una intensa actividad petrolera. Esta región ha sido fuertemente afectada por los derrames de crudo, el sitio ha estado contaminado por los desechos petroleros desde hace décadas y los hidrocarburos han experimentado un proceso de intemperización. En este estudio las muestras de suelo se extrajeron de los primeros $15 \mathrm{~cm}$ de profundidad y se analizaron siguiendo las especificaciones de la NOM-138-SEMARNAT/SSA1-2012. Las muestras contaminadas presentaron textura franco arcillosa, materia orgánica de $6.8 \%$, densidad bacteriana $1.7 \mathrm{x}$ $10^{5}$ de unidades formadoras de colonias (UFC)/g de suelo seco, conductividad eléctrica del suelo (CE) de $0.508 \pm 0.1 \mu \mathrm{S} / \mathrm{cm}$ y un contenido de hidrocarburos de fracción pesada (HFP) de $80926.4 \pm 50 \mathrm{mg} \mathrm{HFP} / \mathrm{kg}$ suelo seco.

\section{Consorcio adaptado}

Previamente a este estudio se evaluó el potencial de biodegradación de dos consorcios microbianos procedentes de sitios contaminados con hidrocarburos desde hace décadas. Después de dos meses de adaptación se utilizó el consorcio microbiano con mayor potencial de biodegradación el cual fue adaptado al $1 \%$ de fracción pesada del crudo Maya y como fuente de nutrientes se empleó Bushnell Haas. Al final del periodo de adaptación el inóculo con mayor potencial de biodegradación tenía una densidad bacteriana de $7 \times 10^{7} \mathrm{UFC} / \mathrm{mL}$. En dicho incóulo fueron identificadas las siguientes especies bacterianas: Flavobacterium salisperosum, Flavobacterium terrae, Flavobacterium saliperosum, Pseudomonas citronellolis, Pseudomonas delhiensis, Pseudomonas knackmussisi, Ochrobacterium intermedium, Ochrobactrum cytisi, Ochrobactrum anthrop y Ochrobactrum tritici, todas con un porcentaje de identidad del $98 \%$.

\section{Evaluación de la actividad microbiana}

La actividad microbiana puede ser expresada en forma de dióxido de carbono $\left(\mathrm{CO}_{2}\right)$ producido, que está correlacionada con la capacidad de biodegradación al ser los hidrocarburos intemperizados la principal fuente de energía. Para la estimación de la actividad microbiana, se utilizó $\mathrm{NaOH}$ para atrapar el $\mathrm{CO}_{2}$ generado de la respiración de los microorganismos. Para lo anterior, se introdujo al frasco que contenía el suelo contaminado un vial con $30 \mathrm{~mL}$ de $\mathrm{NaOH}$, el cual se colocó en otro frasco de $1 \mathrm{~L}$ con 50 $\mathrm{mL}$ de agua para mantener condiciones de humedad. Los frascos fueron guardados a temperatura ambiente y en condiciones de oscuridad para evitar efectos de foto oxidación. Posteriormente, los viales fueron extraídos para su cuantificación a los tiempos de 7,14 y 28 días. Se tomaron alícuotas de $5 \mathrm{~mL}$ de $\mathrm{NaOH}$ y fueron titulados con una solución de $\mathrm{HCl}$ a una concentración de $1 \mathrm{~N}$ y $0.1 \mathrm{~N}$, usando como indicadores fenolftaleína y naranja de metilo, respectivamente. Los análisis se realizaron por duplicado.

\section{Cuantificación de carbono orgánico}

La cantidad de materia orgánica se determinó mediante el método de Walkley y Black (1934), descrito en el apartado AS-07-2000 de la NOM-021-RECANAT-2000. El mecanismo de reacción se basa en la oxidación del carbono orgánico presente en el suelo. El suelo se trató con una solución de dicromato de potasio $\left(\mathrm{K}_{2} \mathrm{Cr}_{2} \mathrm{O}_{7}\right)$, seguida de la adición de ácido sulfúrico $\left(\mathrm{H}_{2} \mathrm{SO}_{4}\right)$ concentrado. A la muestra, enfriada y diluida, se le adicionó ácido fosfórico $\left(\mathrm{H}_{3} \mathrm{PO}_{3}\right)$ y el exceso de ácido crómico $\left(\mathrm{H}_{2} \mathrm{CrO}_{4}\right)$ se tituló con difenilamina-sulfonato de bario como indicador. El contenido de materia orgánica se valora con sulfato ferroso $\left(\mathrm{FeSO}_{4}\right)$. El aumento de biomasa durante el proceso de biodegradación puede ser cuantificado mediante esta técnica. Se ha reportado que los hidrocarburos altamente recalcitrantes no son oxidados durante la reacción exotérmica llevada a cabo entre los compuestos petrolíferos y el $\mathrm{H}_{2} \mathrm{SO}_{4}$, por lo que los resultados derivados de esta técnica deben ser cuidadosamente estudiados y evaluados en conjunto 
con otros análisis, como la determinación de HFP $/ \mathrm{kg}$ suelo para corroborar la disminución de la fracción orgánica correspondiente a los hidrocarburos.

\section{Determinación de las características fisicas y químicas}

Además del monitoreo de la actividad microbiana, se estudiaron otros parámetros físicos y químicos que permitieron identificar las mejores condiciones nutrimentales y que influyeron en el incremento de la biodisponibilidad de los hidrocarburos, alcanzando la máxima biodegradación de los hidrocarburos intemperizados.

El pH se determinó por la norma NOM-021-RECANAT-2000. Se utilizó un potenciómetro modelo "Oakton $\mathrm{pH} / \mathrm{Conductivity} / \mathrm{TDS} /{ }^{\circ} \mathrm{C} /{ }^{\circ} \mathrm{F}$ Meter" con una relación suelo-agua de 1:2. La determinación de la humedad se realizó conforme a la NOM-021-SEMARNAT-2000, la cual establece su método en el apartado AS-06. Se empleó un equipo de secado marca "Sartorius MA 35", a partir de una muestra de $0.5 \mathrm{~g}$ y se registró la humedad inicial. La conductividad eléctrica del suelo contaminado se determinó mediante la NOM-021-RECANAT-2000, por el método AS18-2000. Para ello, se añadió agua desionizada a una muestra de suelo, se agitó y posteriormente se dejó reposar. La conductividad eléctrica del sobrenadante se estimó con un conductímetro "Hach TDS Meter". La determinación de los HFP se realizó siguiendo las especificaciones de la norma NMX-AA-145-SFCI-2008.

\section{Diseño de experimentos}

En el proceso de biodegradación se debe considerar todas las fuentes de nutrientes que pueden ser empleadas por los microorganismos. Por ejemplo, aquellos que interactúan mediante uniones físicas y químicas a la fracción mineral del suelo y la materia orgánica o los nutrientes presentes en compuestos agroindustriales. Todas estas fuentes pueden actuar como medios para obtener nutrientes y ser utilizados en los procesos metabólicos de los microorganismos. El diseño de Plackett-Burman es un diseño de barrido que establece relaciones entre los factores de estudio y la variable de respuesta. Permite reducir el número de experimentos considerablemente al tratarse de un modelo ortogonal (Montgomery 1991). El diseño de experimentos consiste en 11 factores, que fueron las sales inorgánicas estudiadas en 12 tratamientos. El experimento consideró dos réplicas para llevar a cabo el análisis estadístico.

Los dos niveles de concentración para el diseño de experimentos fueron: nivel alto (1) y nivel bajo (0) como se muestra en el cuadro I. La concentración

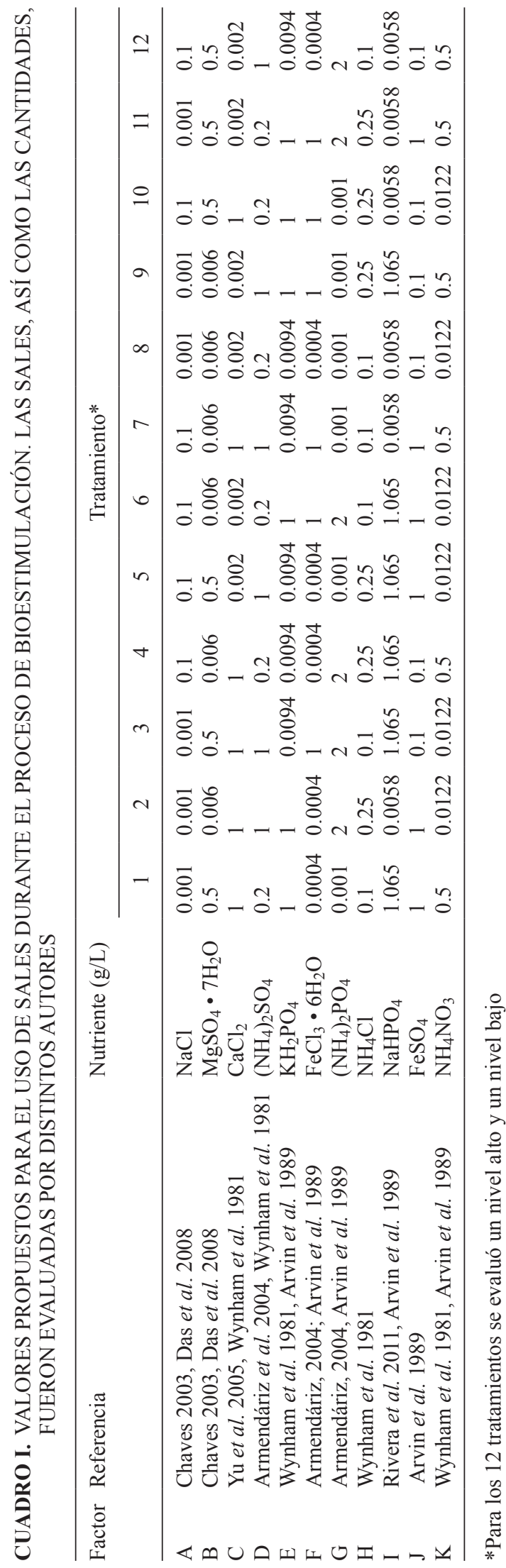


de sales se estableció con base en lo reportado por otros autores en la biodegradación de hidrocarburos persistentes al ser los compuestos que aportan los nutrientes necesarios para promover la actividad microbiana eficientemente.

\section{Configuración del proceso de biodegradación a nivel laboratorio}

El estudio de la biodegradación de los hidrocarburos intemperizados se llevó acabo en 12 tratamientos simulando un ambiente húmedo dentro de un recipiente de vidrio de $1 \mathrm{~L}$ al añadir $50 \mathrm{~mL}$ de agua destilada bajo condiciones de oscuridad y temperatura ambiente. Adicionalmente se introdujo al frasco de $1 \mathrm{~L}$, otro frasco de vidrio con $30 \mathrm{~g}$ de suelo contaminado más un vial con $30 \mathrm{~mL}$ de $\mathrm{NaOH}$ para capturar el $\mathrm{CO}_{2}$ producido durante la actividad microbiana. A cada frasco con el suelo se adicionó $1 \%$ del consorcio adaptado. El consorcio se adaptó en $270 \mathrm{~mL}$ de medio Bushnell Haas por dos meses, el inóculo se incubó a $28^{\circ} \mathrm{C}$ con agitación a $200 \mathrm{rpm}$ con un agitador marca "Brunswick modelo C-25". Cada tratamiento contenía microbiota nativa en una concentración al $1 \times 10^{3} \mathrm{UFC} / \mathrm{g}$ con excepción de los testigos positivo $(\mathrm{P})$ y negativo $(\mathrm{N})$. El primero consistió en un suelo contaminado sin la adición de nutrientes y agua, mientras que el segundo fue ladrillo molido incinerado a una temperatura de $600{ }^{\circ} \mathrm{C}$.

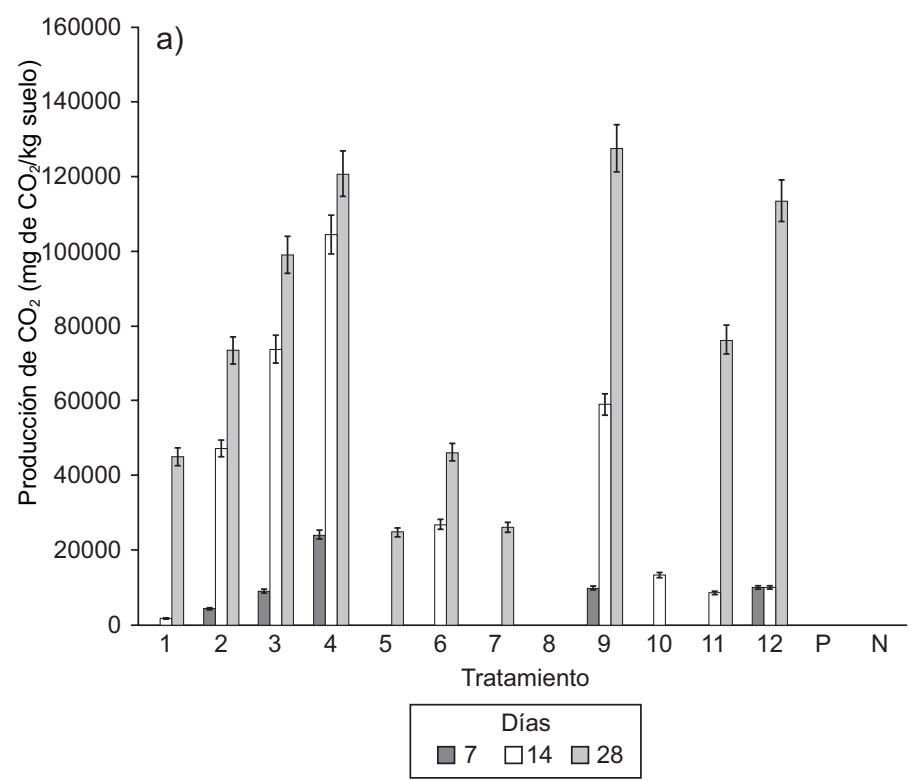

Asimsimo, se realizó otro testigo que consistió en preparar bajo las mismas condiciones experimentales un suelo estéril con la adición de nutrientes para observar algún posible efecto físico o químico ocasionado por las sales inorgánicas adicionadas al suelo contaminado.

\section{Análisis estadístico}

El análisis estadístico consideró dos réplicas, se evaluó la significancia de cada factor en las variables de estudio empleando el programa Statgraphics 15.0 con una $\mathrm{P}<0.05$.

\section{RESULTADOS Y DISCUSIÓN}

\section{Respiración microbiana}

Se estimó la actividad microbiana (expresada como $\mathrm{mg} \mathrm{CO} / 2 \mathrm{~kg}$ suelo seco) que fue llevada a cabo por los microorganismos para los 12 tratamientos. La figura 1a muestra la producción de $\mathrm{CO}_{2}$ para los 12 tratamientos a los 7, 14 y 28 días de biodegradación. Esta medición es un parámetro indirecto para medir la capacidad de degradación de los microorganismos hidrocarbonoclastas (Anderson 1982, D'Annibale et al. 2004). Se observó un aumento progresivo de la actividad microbiana en los tratamientos 4,9 y 12 con un valor de 132180,94246 y $92107 \mathrm{mg} \mathrm{CO}_{2} / \mathrm{kg}$ suelo

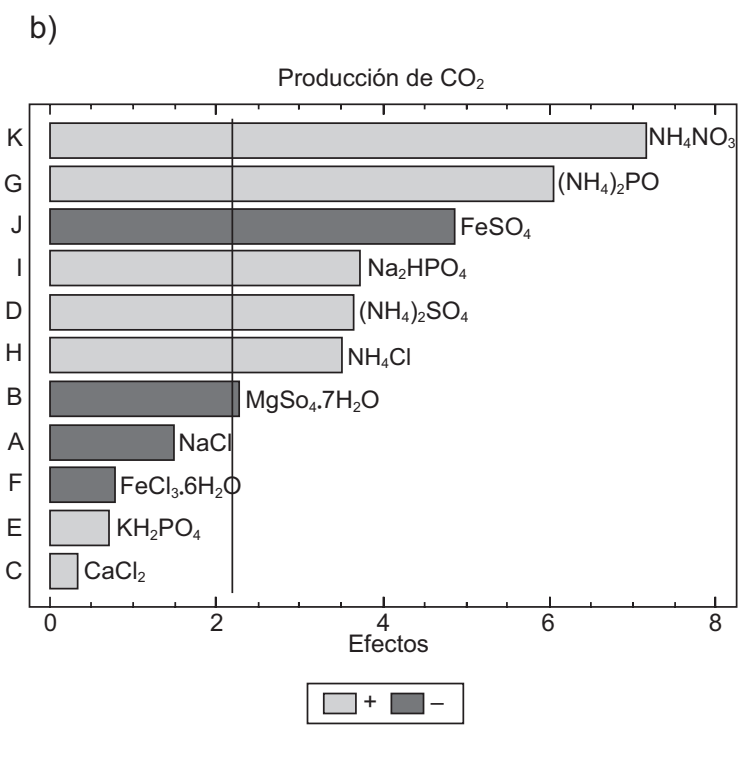

Fig. 1. Producción de $\mathrm{CO}_{2}$ : (a) Estimación de la producción de $\mathrm{CO}_{2}$ a los 7, 14 y 28 días de la degradación de los hidrocarburos intemperizados del suelo, las barras de error muestran la desviación estándar de la generación de $\mathrm{CO}_{2}$ de los 12 experimentos a partir de dos réplicas, (b) Diagrama de Pareto donde se muestran únicamente los factores (A-K) correspondientes a las sales minerales que resultaron ser más significativos en la producción de $\mathrm{CO}_{2}$. El análisis de varianza mostró los valores de $\mathrm{R}^{2}=$ $93.5 \%, \mathrm{R}_{\text {ajustada }}=87.6 \%$ y un error estándar $=16963$ de todos los experimentos. La producción de $\mathrm{CO}_{2}$ en el suelo tratado se comparó con dos suelos: un testigo positivo $(\mathrm{P})$ y un testigo negativo $(\mathrm{N})$ 
a los 28 días, respectivamente, siendo el tratamiento 9 el de mayor producción de $\mathrm{CO}_{2}$. El diagrama de Pareto (Fig. 1b) muestra las sales inorgánicas NH${ }_{4} \mathrm{NO}_{3}$ y $\left(\mathrm{NH}_{4}\right)_{2} \mathrm{PO}_{4}$, consideradas estadísticamente significativas durante la respiración microbiana. Tales compuestos intervinieron de manera importante con un nivel alto en la producción de $\mathrm{CO}_{2}$ y por consiguiente en la actividad metabólica de los microorganismos presentes en el suelo. El nitrógeno y potasio influyen en la formación de nuevas células, intervienen en los procesos cometabólicos y en la formación de productos intermediarios específicos como los ácidos grasos y los ácidos carboxílicos, los cuales serán asimilados por otras especies microbianas.

La producción de $\mathrm{CO}_{2}$ fue nula para los controles $\mathrm{Py} \mathrm{N}$, al igual que en el tratamiento 8 . Esto sugiere que la aplicación de nutrientes y su concentración son determinantes para incrementar la actividad microbiana.

\section{pH}

El pH en los suelos costeros afecta la solubilización de los nutrientes y de los hidrocarburos persistentes. Los suelos contaminados con hidrocarburos tienden a ser suelos ácidos, sin embargo, para que la biodegradación sea llevada a cabo se requiere un $\mathrm{pH}$ neutro entre 7.4 y 7.8. Los tratamientos que se encuentraron dentro de este rango de $\mathrm{pH}$ a los 28 días de biodegradación fueron: 3, 4, 6, 9 y 10 (Fig. 2a). Esto demuestra que el tipo de nutriente y su concentración influyen en la alteración de las propiedades fisicas y químicas. El cambio de $\mathrm{pH}$ ligeramente ácido a neutro favorece los procesos metabólicos de las especies microbianas involucradas en la degradación de hidrocarburos (Arrieta 2011). La formación de subproductos ocasiona cambios de $\mathrm{pH}$ durante el proceso de biodegradación de los hidrocarburos. Las especies iónicas formadas en ocasiones tienden a inhibir la actividad microbiana por la formación de metabolitos que ocasionan un ambiente tóxico en la fase líquida del suelo, lo que disminuye el crecimiento de bacterias hidrocarbonoclastas, o bien ciertos subproductos de la biodegradación pueden ser empleados como fuente de energía por otras especies que no participan en la degradación del contaminante. De acuerdo con el diagrama de Pareto las sales que mostraron mayor significancia en la alteración del pH son: $\mathrm{KH}_{2} \mathrm{PO}_{4}$, $\left(\mathrm{NH}_{4}\right)_{2} \mathrm{PO}_{4}, \mathrm{MgSO}_{4} \cdot 7 \mathrm{H}_{2} \mathrm{O}, \mathrm{FeSO}_{4}$ (Fig. 2b). Estas sales contienen fósforo en forma de fosfato $\left(\mathrm{PO}_{4}\right)$, compuesto que participa en la síntesis síntesis de enzimas. El pH afecta la solubilidad de los nutrientes y el transporte de metales traza o metales pesados presentes en el suelo (Riser-Roberts 1998).

b)

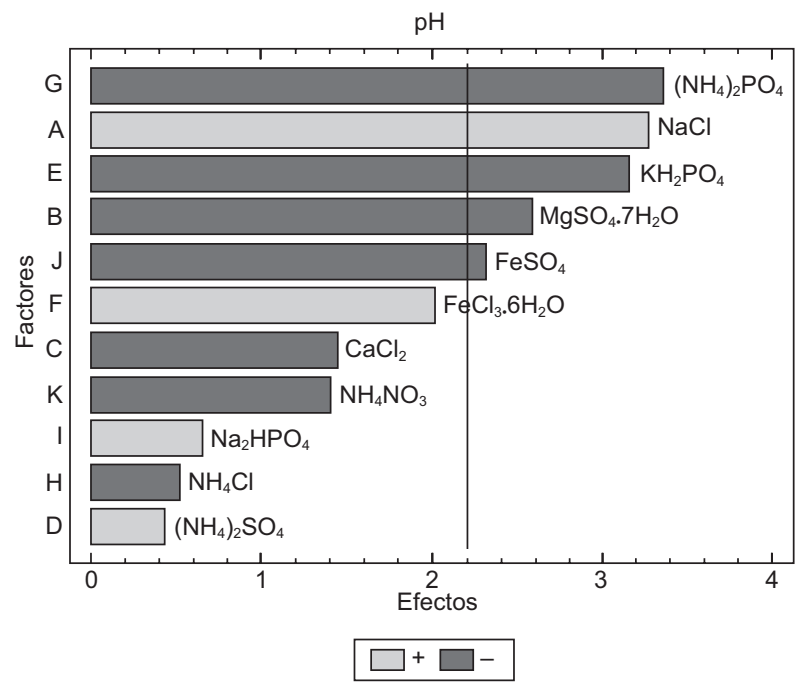

Fig. 2. Medición de pH: (a) Estimación del pH para los 12 tratamientos durante la degradación de los hidrocarburos intemperizados durante 28 días, así mismo, las líneas de error representan la desviación estándar de cada uno de los tratamientos a partir de dos réplicas. Los valores 1 a 12 corresponden a los experimentos, en tanto, $\mathrm{P}$ y $\mathrm{N}$ corresponden a los testigos positivo y negativo, (b) Diagrama de Pareto donde se muestran únicamente los factores (A-K) que son significativos en el cambio de $\mathrm{pH}$ durante de los 28 días de biodegradación. El análisis de varianza mostró los valores de $\mathrm{R}^{2}=83.6 \%, \mathrm{R}_{\mathrm{ajustada}}=68.6 \%$ y el error estándar $=0.22$ 
Es posible favorecer el cambio del $\mathrm{pH}$ al mantener la concentración mínima necesaria de nutrientes esenciales en el suelo y generar subproductos específicos, como ácidos carboxílicos, alcoholes y ésteres. Estos pueden determinar un ambiente ácido o alcalino en la fase líquida e influir en el desarrollo de especies microbianas capaces de degradar los hidrocarburos adsorbidos en los poros del suelo. Dias et al. (2012) han reportado relaciones de nutrientes esenciales $\mathrm{C}: \mathrm{N}: \mathrm{P}(100: 10: 1)$ en la fase líquida del suelo que mejoran el $\mathrm{pH}$, es decir que lo incrementan de 5 a 7 y con ello, se favorece un crecimiento microbiano eficiente que promueve la biodegradación de los hidrocarburos intemperizados. Por consiguiente, se deben de considerar todas las fuentes de nutrientes que afectan los procesos metabólicos así como el tipo de fracción mineral o arcilla y los contaminantes presentes en el suelo. En cuanto a los nutrientes en la fracción mineral, durante la biodegradación hay componentes iónicos que, al ser desadsorbidos debido a los subproductos desarrollados, pueden ser bioasimilados. Por otro lado, los nutrientes derivados de fuentes antrópicas, como los fertilizantes en el suelo, también contribuyen a la concentración de nutrientes que pueden ser aprovechados por los microorganismos. Conocer el tipo y concentración de nutrientes necesarios a introducir en un suelo contaminado para obtener una eficiente biodegradación implica altos costos y tiempo de investigación (Riser-Roberts 1998).

\section{Porcentaje de humedad}

La humedad del suelo es un factor limitante para el éxito de la biorremediación. Previamente a este estudio se evaluaron diferentes porcentajes de humedad en la biodegradación de los hidrocarburos. Se obtuvo que una humedad del $30 \%$ tiene una mayor influencia en la biodegradación de hidrocarburos intemperizados. Por la razón anterior es que en el tiempo cero todos los experimentos comenzaron con una humedad fija del $30 \%$. Posteriormente durante el periodo de biodegradación de los hidrocarburos, la humedad presentó aumentos o decrementos lo que se asocia a la biodegradación de los hidrocarburos y no por efectos abióticos puesto que los experimentos se desarrollaron en la oscuridad, bajo condiciones herméticas y controladas para evitar pérdidas por volatilización o foto oxidación. Los porcentajes de humedad para cada tratamiento se observan en la figura 3a. En todos los tratamientos hubo un aumento de la humedad en el día 7, en un intervalo del 10 al $20 \%$ para los tratamientos, lo que se atribuye a la mineralización de compuestos hidrocarbonados debido a la formación de $\mathrm{CO}_{2}$ y agua. Asimsimo, la producción

b)

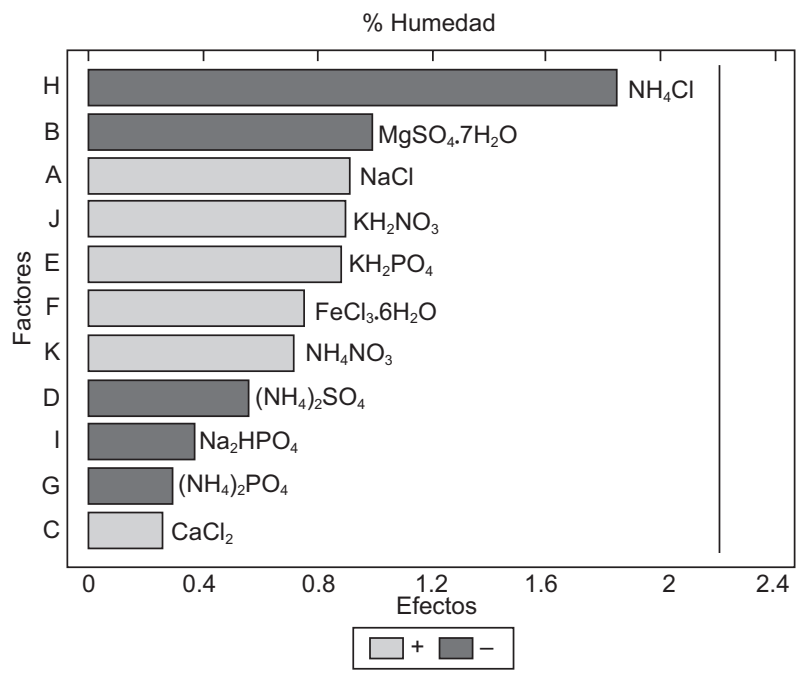

Fig. 3. Humedad relativa (HR): (a) Estimación del porcentaje de humedad de los 12 experimentos durante los 28 días de la biodegradación de los hidrocarburos intemperizados, así mismo, las líneas de error representa la desviación estándar de cada experimento en dos réplicas. Los valores 1 a 12 corresponden a los experimentos, en tanto, $\mathrm{P}$ y $\mathrm{N}$ son los testigos positivo y negativo, (b) Diagrama de Pareto donde se muestran únicamente los factores (A-K) que resultaron ser más significativos para el cambio de la humedad del suelo en el proceso de biodegradación. El análisis de varianza mostró los valores de $\mathrm{R}^{2}=90.0 \%, \mathrm{R}_{\mathrm{ajustada}}=81.3 \%$ y el error estándar $=2.5$ 
de nuevos subproductos incrementa la solubilidad de los compuestos orgánicos por lo que la tasa de biodegradación es directamente proporcional a la humedad en un sistema cerrado (Place et al. 2001). Después del día 14 se observó el mayor aumento de la humedad en los tratamientos $1,3,4,6$ y 9 , respecto a los demás. Los testigos Py N presentaron menor contenido de humedad respecto a los demás tratamientos. El incremento de humedad está correlacionado con la biodegradación de los hidrocarburos, siendo el agua parte de los subproductos obtenidos. Asimismo, muchos subproductos como algunos gases provenientes de la biodegradación, como el $\mathrm{CO}_{2}$ y el $\mathrm{H}_{2} \mathrm{O}$, alteran las propiedades fisicas y químicas del suelo debido al incremento en la presión interna de su matriz. Esto ocasiona un incremento en la porosidad (Place et al. 2001), lo que permite un mayor transporte de agua, nutrientes, oxígeno y microorganismos entre los poros del suelo. Los tratamientos y los testigos $\mathrm{P}$ y $\mathrm{N}$ presentaron actividad microbiana nula debido a la falta de nutrientes. Cuando no existen las condiciones de humedad y de nutrientes en el suelo, el tamaño de cada microorganismo disminuye. Por lo que estos microorganismos que ocupan los poros del suelo son reemplazados por hidrocarburos hidrofóbicos que quedan secuestrados entre dichos espacios porosos. El diagrama de Pareto no muestra un efecto signifi- cativo de los nutrientes sobre la humedad (Fig. 3b). Sin embargo, la humedad controla el transporte de nutrientes entre el suelo y la pared celular de los microorganismos al existir un mayor contacto y su correspondiente asimilación. En suelos de granulometría fina como los arcillosos y franco-arcillosos suele haber un rango óptimo de humedad cercano al $40 \%$ de la capacidad de campo para la biodegradación de contaminantes (Rehm et al. 2000). No obstante, cuando existe un exceso de agua en los suelos, además de anegarse, obtienen una textura viscosa por la mezcla de esta agua con sus contenidos arcillosos. Lo que interfiere con el transporte de oxígeno entre los poros del suelo, modificando así el entorno hacia condiciones anaeróbicas (Arrieta 2011).

\section{Conductividad eléctrica (CE)}

La capacidad de degradación de los hidrocarburos estuvo asociada con los cambios en la CE. Esta es un parámetro que es afectado por la concentración de iones solubles tales como $\mathrm{Ca}^{2+}, \mathrm{Mg}^{2+}, \mathrm{K}^{+}$y Na${ }^{+}$, los que interfieren en el crecimiento de los microorganismos. En la figura 4a se observa que para el día 14 se duplicó el valor de la CE respecto al día 7, para la mayoría de los tratamientos. En el día 28 el valor de $\mathrm{CE}$ más alto fue para el tratamiento 9 , con $0.94 \mu \mathrm{S} / \mathrm{cm}$. En tanto para la mayoría de los tratamientos la CE

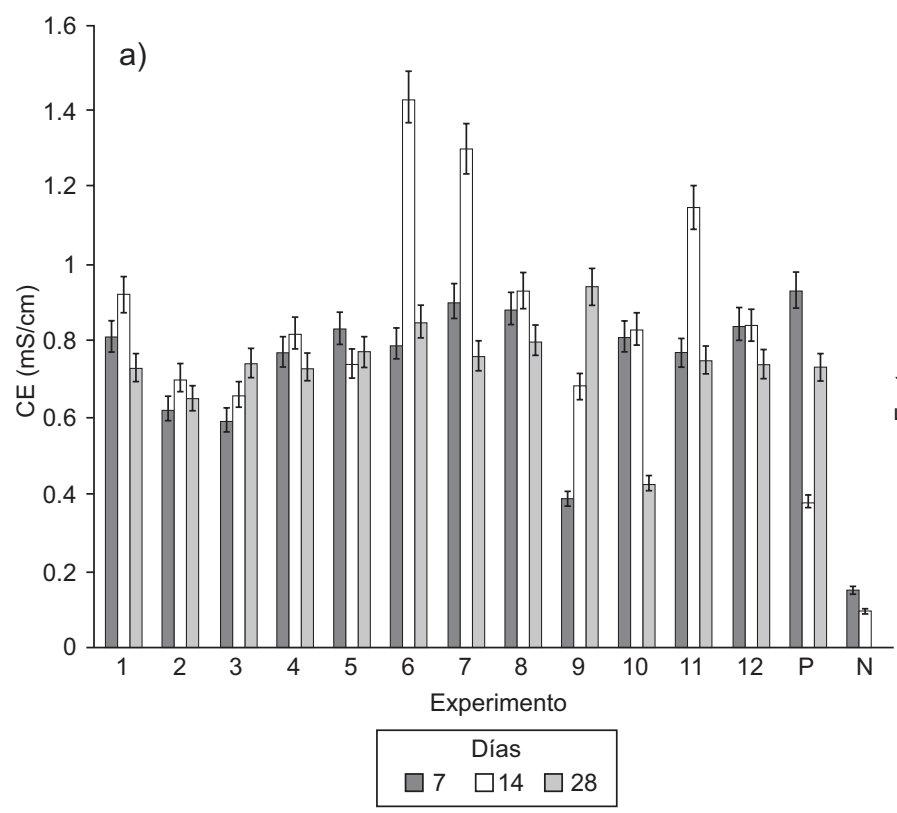

b)

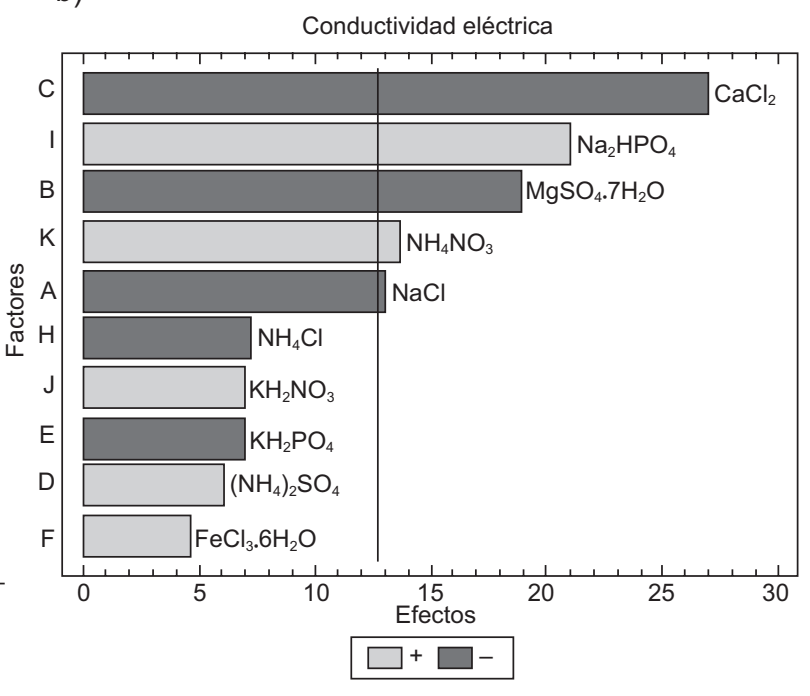

Fig. 4. Conductividad eléctrica (CE): (a) Estimación de CE de los 12 experimentos durante 28 días de biodegradación de los Fig. intemperizados, así mismo, las líneas de error representan la desviación estándar de cada experimento en dos réplicas. Los valores 1 a 12 corresponden a los experimentos, en tanto, $\mathrm{P}$ y N son los testigo positivo y negativo, (b) Diagrama de Pareto donde se muestran únicamente los factores (A-K) que resultaron ser más significativos para el cambio de la CE del suelo en el proceso de biodegradación. El análisis de varianza mostró los valores de $\mathrm{R}^{2}=93.5 \%, \mathrm{R}_{\text {ajustada }}=87.6 \%$ y el error estándar $=0.05$ 
disminuyó a los 14 y 28 días. A nivel del campo la $\mathrm{CE}$ del suelo modifica con el paso del tiempo debido a la acumulación de compuestos ácidos o alcalinos. Un pH bajo tiende a solubilizar las especies iónicas durante un proceso de biodegradación influenciado por la generación de subproductos lo que ocasiona un aumento en los valores de CE (Acar et al. 1996). Algunos autores han encontrado en los suelos restaurados que los niveles de $\mathrm{N}$ y $\mathrm{P}$ disminuyen hasta tres veces, por lo que se incrementa dos veces la $\mathrm{CE}$ neutro o ligeramente alcalino (Trujillo et al. 2012).

Dado lo anterior, en un proceso de bioestimulación es determinante considerar con precisión el papel de cada nutriente para alcanzar la máxima biodegradación (García 2012). Normalmente los suelos costeros contaminados con hidrocarburos intemperizados tienen bajas cantidades de nutrientes por efecto de la lixiviación. Un incremento de iones en el suelo inhibe la degradación de los nutrientes o al haber un exceso de ellos se puede ocasionar la eutrofización por la aparición de algas y otros microorganismos que promueven los procesos anaeróbicos (Schroeder et al. 1999).

En la figura $4 \mathbf{b}$ el diagrama de Pareto muestra que nutrientes como el sulfato de magnesio $\left(\mathrm{MgSO}_{4}\right)$ tienen una mayor influencia en el aumento de la CE. y cuatro veces el $\mathrm{Na}$, lo que altera el $\mathrm{pH}$ de ácido a

Asimismo, se encontró que esta sal tiene influencia importante en otros parámetros analizados como el $\mathrm{pH}$ y el porcentaje de humedad, aún en pequeñas cantidades, es estadísticamente significativo.

El Mg está presente regularmente en suelos arcillosos, por lo que la alteración de las propiedades fisicas y químicas del suelo como la CE, la humedad y el pH están directamente relacionadas con la presencia de este elemento (Hernández 2003).

\section{Materia orgánica}

La figura 5a muestra que los tratamientos 5,9 y 10 tienen menor contenido de materia orgánica una vez transcurridos los 28 días de biodegradación con porcentajes de $3.78 \%, 2.17 \%$ y $2.54 \%$ respectivamente. Algunos autores han asociado la disminución del contenido de materia orgánica en el suelo con la degradación de la fuente de la materia hidrocarbonada (Ramírez 2009).

Mediante el método de "Walkley and Black", las cadenas cortas de hidrocarburos $\left(\mathrm{C}_{5}\right.$ a $\left.\mathrm{C}_{9}\right)$ se volatilizan por la reacción exotérmica con el $\mathrm{H}_{2} \mathrm{SO}_{4}$, mientras que el resto se oxida. Cadenas más largas $\left(\mathrm{C}_{10}\right.$ a $\left.\mathrm{C}_{23}\right)$ tienen menor cantidad de compuestos volátiles, al oxidarse se estiman valores altos de materia orgánica. Sin embargo, los hidrocarburos con cadenas más largas

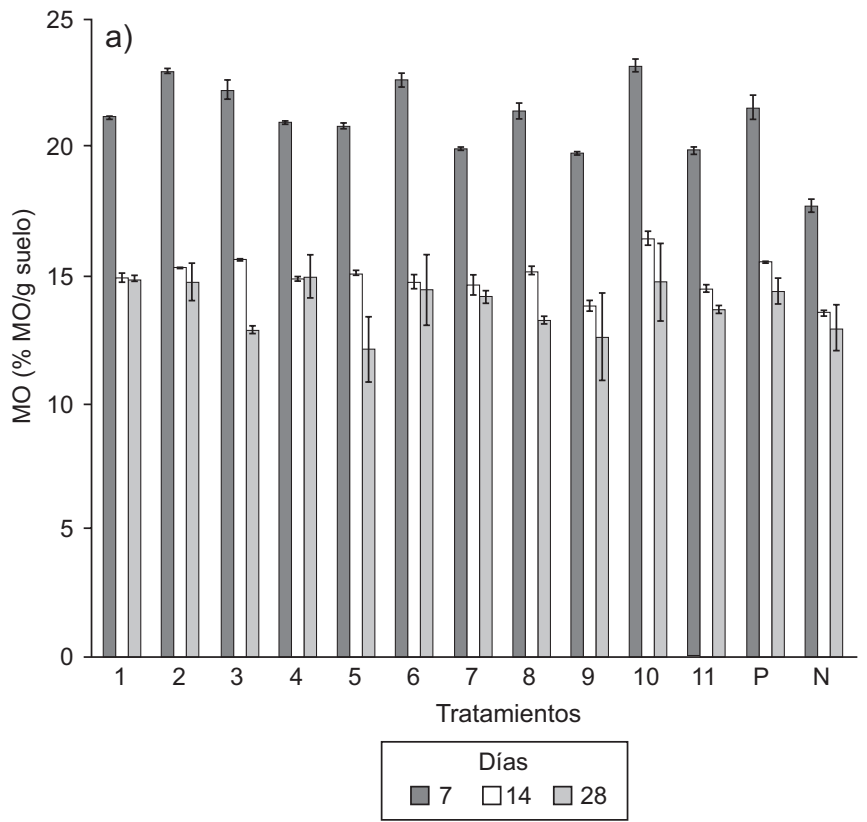

b)

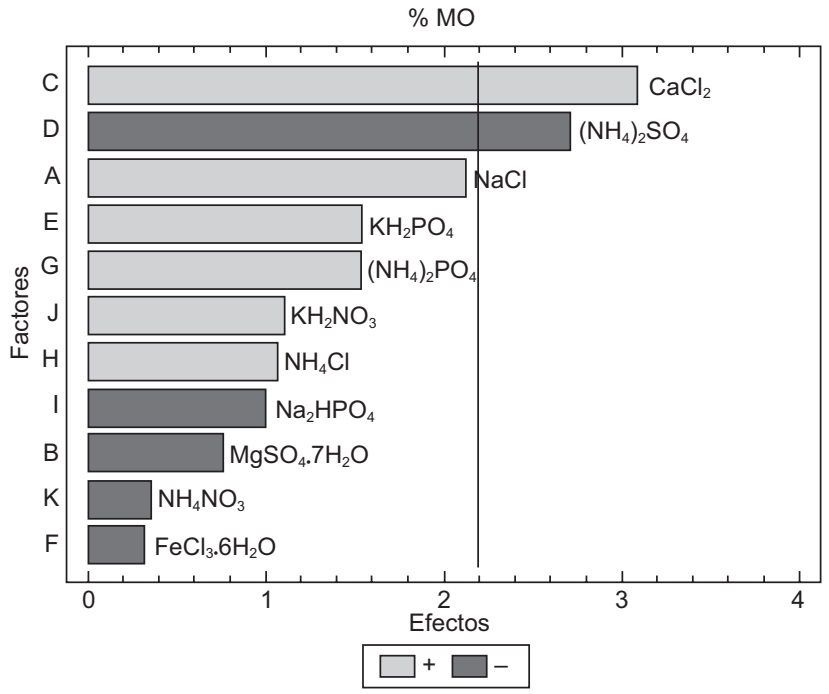

Fig. 5. Materia orgánica (MO): (a) Estimación del porcentaje de MO de los 12 experimentos durante 28 días de la biodegradación de los hidrocarburos intemperizados, así mismo, las líneas de error representan la desviación estándar de cada experimento en dos réplicas. Los valores 1 a 12 corresponden a los experimentos, en tanto, $\mathrm{P}$ y $\mathrm{N}$ corresponden a los testigos positivo y negativo, (b) Diagrama de Pareto donde se muestran únicamente los factores (A-K) que resultaron ser más significativos para la estimación del porcentaje de la materia orgánica. El análisis de varianza mostró los valores de $\mathrm{R}^{2}=75.9 \%$, $\mathrm{R}_{\text {ajustada }}=53.9 \%$ y el error estándar $=0.78$ 


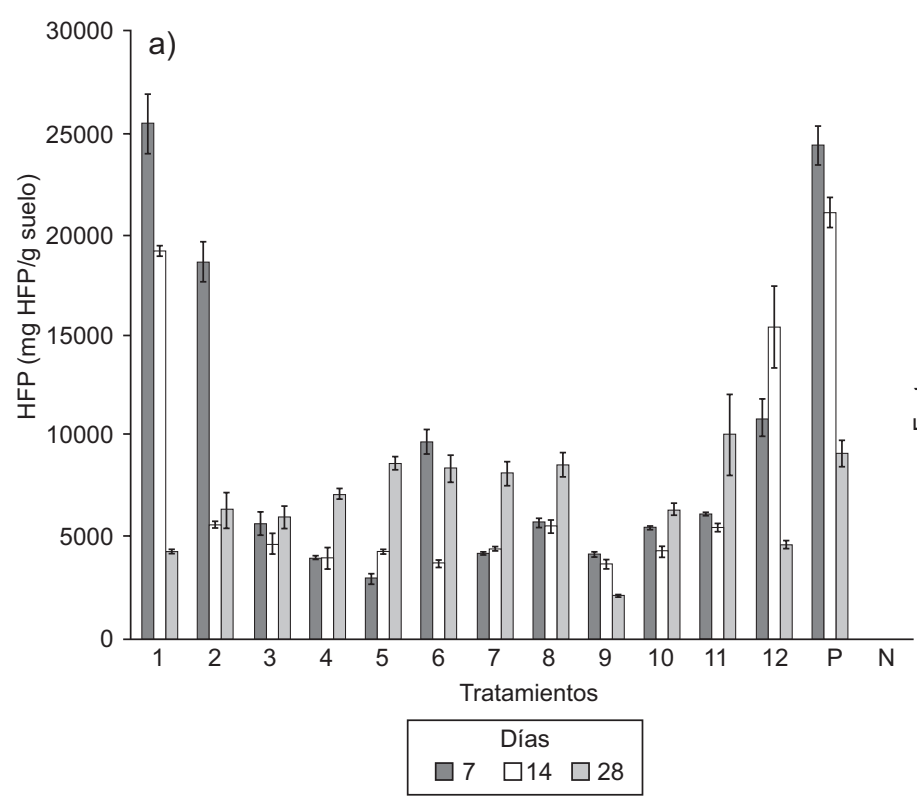

b)

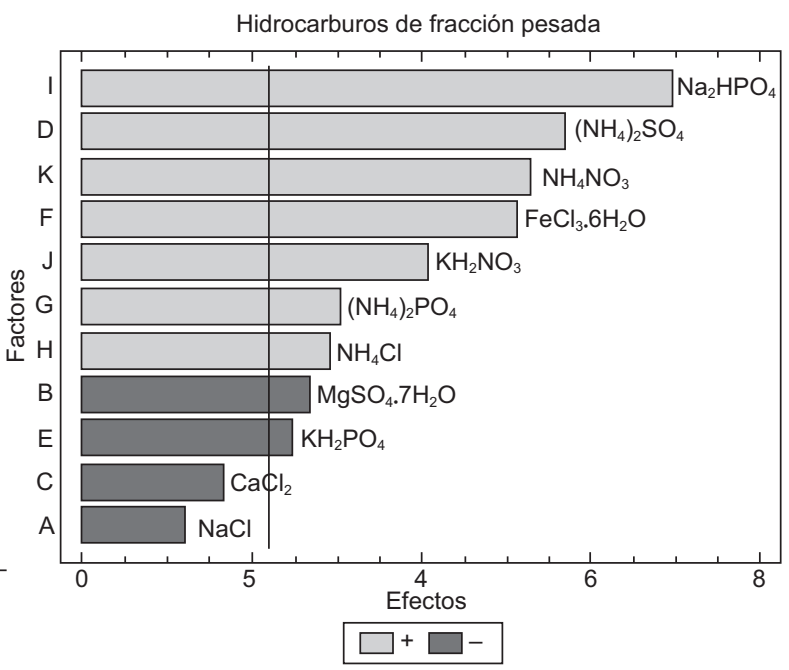

Fig. 6. Hidrocarburos de fracción pesada (HFP): (a) Estimación del contenido de HFP para los 12 tratamientos durante 28 días de la biodegradación de los hidrocarburos intemperizados, así mismo, las líneas de error representan la desviación estándar de cada experimento en dos réplicas. Los valores 1 a 12 corresponden a los experimentos, en tanto, $\mathrm{P}$ y $\mathrm{N}$ corresponden a los testigos positivo y negativo, (b) Diagrama de Pareto donde se muestran únicamente los factores (A-K) que son más significativos para la medición del contenido de HFP. El análisis de varianza mostró los valores de $\mathrm{R}^{2}=94.5 \%$, $\mathrm{R}_{\text {ajustada }}=89.46 \%$ y un error estándar $=1108$

$\left(\mathrm{C}_{25}\right.$ a $\left.\mathrm{C}_{35}\right)$, son difíciles de volatilizar por este método, ya que sólo una pequeña parte de ellos son oxidables. En el diagrama de Pareto (Fig. 5b) se observa que la mayoría los nutrientes no influyeron significativamente en el aumento del porcentaje de materia orgánica. No obstante, sí influyeron en el proceso metabólico de los microorganismos, todo depende de que tan asimilables sean para ellos, lo cual va a estar determinado por las condiciones ambientales, las características de la microbiota y el tipo de contaminantes.

\section{Hidrocarburos de fracción pesada (HFP)}

En la figura 6a se muestran las concentraciones de los HFP de los 12 tratamientos. Los testigos P y $\mathrm{N}$, así como los tratamientos 1 y 2 son los que presentaron mayor contenido de los HFP a los 7 días de biodegradación. La disminución en la concentración de los HFP se atribuyó a los nutrientes aplicados y al consorcio microbiano adaptado. Se observó relación entre la producción de $\mathrm{CO}_{2}$ y la degradación de la fracción pesada, ya que el experimento 1 registró menor producción de $\mathrm{CO}_{2}$ y también mayor contenido de los HFP a los 7 y 14 días de biodegradación. En tanto que el tratamiento 9 presentó la menor concentración de hidrocarburos con una concentración de $3775 \mathrm{mg} \mathrm{HFP} / \mathrm{kg}$ suelo seco, además fue el de mayor producción de $\mathrm{CO}_{2}$. La figura $6 \mathbf{b}$ muestra que la mayoría de los nutrientes influyeron significativamente en la degradación de los HFP. Una vez evaluadas las características fisicas y químicas del suelo se concluyó que el tipo y concentración de cada sal influyeron en la biodegradación de los hidrocarburos, siendo el tratamiento 9 el que presentó una mejor respuesta a la degradación de los compuestos hidrocarbonados.

\section{CONCLUSIONES}

La concentración mínima necesaria de nutrientes esenciales es un factor limitante en la biotransformación de hidrocarburos intemperizados. Estos hidrocarburos no pueden ser degradados por la microbiota nativa únicamente debido a la carencia de un sistema enzimático específico y a la recalcitrancia de los compuestos. Se demostró que la aplicación del tipo y concentración de sales inorgánicas, así como la adición al suelo contaminado de un consorcio adaptado con hidrocarburos de fracción pesada, incrementan la capacidad de biodegradación de los hidrocarburos persistentes. El diseño Plakett Burman permitió identificar las mejores condiciones nutrimentales, mismas que corresponden a la formulación 9: (g/L) $0.001 \mathrm{NaCl}, 0.006 \mathrm{MgSO}_{4} \cdot 7 \mathrm{H}_{2} \mathrm{O}, 0.002$ 
$\mathrm{CaCl}_{2}, 1\left(\mathrm{NH}_{4}\right)_{2} \mathrm{SO}_{4}, 1 \mathrm{KH}_{2} \mathrm{PO}_{4}, 1 \mathrm{FeCl}_{3} \cdot 6 \mathrm{H}_{2} \mathrm{O}$, $0.001\left(\mathrm{NH}_{4}\right)_{2} \mathrm{PO}_{4}, 0.25 \mathrm{NH}_{4} \mathrm{Cl}, 1.065 \mathrm{NaHPO}_{4}, 0.1$ $\mathrm{FeSO}_{4}, 0.5 \mathrm{NH}_{4} \mathrm{NO}_{3}$.

Este proyecto sirve como una contribución al mejoramiento de tecnologías de bioestimulación con el objetivo de promover la proliferación de microorganismos hidrocarbonoclastas capaces de degradar hidrocarburos altamente persistentes.

\section{REFERENCIAS}

Acar Y.B. y Alshawabkeh A.N. (1996). Electrokinetic remediation. I: Pilot-scale tests with lead-spiked kaolinite. J. Geotech. Geoenviron. 122, 173-185.

DOI: 10.1061/(ASCE)0733-9410(1996)122:3(173)

Adams R.H., Olán-Castro D., Guzmán-Osorio F.J. y DíazRamírez I.J. (2009). Relationship between gemorphology and contamination with weathered hydrocarbon in an old river levee/marsh association. Int. J. Environ. Sci. Tech. 6, 527-538.

DOI: $10.1007 / \mathrm{BF} 03326092$

Anderson J.P.E. (1982). Soil respiration. En: Methods of soil analysis. (A.L Page, R.H. Miller, D.R. Keeney, Eds.). Part II. Chemical and microbiological properties. American Society of Agronomy, Madison, Wisconsin, EUA, pp. 831-871.

Antizar-Ladislao B., López-Real J.M. y Beck A.J. (2006). Investigation of organic matter dynamics during invessel composting of aged coal tar contaminated soil by fluorescence excitation-emission spectroscopy. Chemosphere 64, 839-847.

DOI: $10.1016 /$ j.chemosphere.2005.10.036

Arrieta R.O. (2011). Evaluación de la influencia del bioestímulo sobre un suelo contaminado con diesel y su integración a la gestión ambiental. Tesis de Maestría. Universidad Nacional de Colombia. Medellín, Colombia, 123 pp.

Armendáriz P., Loera-Corral O., Fernández-Linares L., Esparza-García F. y Rodríguez Vázquez R. (2004). Biostimulation of micro-organims from sugarcane bagase pith for the removal of weathered hydrocarbon from soil. Lett. Appl. Microbiol. 38, 372-377.

DOI: $10.1111 / \mathrm{j} .1472-765 X .2004 .01502 . \mathrm{x}$

Arvin E., Jensen B.K. y Gundersen A.T. (1989). Substrate interactions during aerobic biodegradation of benzene. Appl. Environ. Microb. 55, 3221-3225.

Place M.C., Coonfare C.T., Chen A.S.C., Hoeppel R.E. y Rosansky S.H. (2001). Principles and practices of bioslurping. Battelle Press.California, EUA, 190 pp.

Chávez-Gómez B., Quintero R., Esparza-García F., Mesta-Horward A.M., Zavala Díaz de la Serna F.J., Hernández-Rodríguez C.H., Gillén T., PoggiVaraldo H.M. y Cortés-Barrera J. (2003). Removal phenanthrene from soil by co-cultures of bacteria and fungi pregrown on sugarcane bagasse pith. Bioresour. Technol. 89, 177-83.

DOI: $10.1016 / \mathrm{S} 0960-8524(03) 00037-3$

D'Annibale A., Ricci M., Leonardi V., Quaratino D., Mincione E. y Petruccioli M. (2004). Degradation of aromatic hydrocarbons by white-rot fungi in a historically contaminated soil. Biotechnol. Bioeng. 90, 6 . DOI: 10.1002/bit.20461

Das P. y Luyan Z. (2012). Pyocyanin pigment assisting biosurfactant-mediated hydrocarbon emulsification. Int. Biodeter Biodegr. 85, 278-283. DOI: $10.1016 /$ j.ibiod.2013.07.013

Das P., Mukherjee S. y Sen R. (2008). Improved bioavailability and biodegradation of a model polyaromatic hydrocarbon by a biosurfactant producing bacterium of marine origin. Chem. 72, 1229-1234.

DOI: 10.1016/j.chemosphere.2008.05.015

Dias R.L., Ruberto L., Hernández E., Vázquez S.C., Lo Balbo A., Del Panno M. T. y Marc Cormack W. P. (2012). Bioremediation of an aged diesel oil-contaminated antartic soil: Evaluation of the "on site" bioestimulation strategy using different nutrient sources. Int. Biodeter. Biodegr. 75, 96-103.

DOI: $10.1016 /$ j.ibiod.2012.07.020

García A.M.C (2012). Rehabilitación de un suelo con bajo perfil de nutrientes aplicando biosólidos como fertilizante. Tesis de maestría, Instituto Politécnico Nacional, Ciudad de México, México, 189 pp.

García R. (2003). Transferencia de masa y biodegradación de hidrocarburos de un suelo intemperizado en un cultivo de suelo en suspensión. Tesis de doctorado, Universidad Autónoma Metropolitana, Ciudad de México, México, $155 \mathrm{pp}$.

INAFED (2010). Enciclopedia de los municipios y delegaciones de México. Veracruz de Ignacio de la Llave. Medio físico: información de las características fisiográficas del estado de Veracruz. Instituto para el Federalismo y Desarrollo Municipal [en línea]. http:// inafed.gob.mx/work/enciclopedia/EMM30veracruz/ index.html

Hernández J., Orihuela D., Pérez-Mohedano S., Marijuan L., Furet N., Madrid E. H.-T. S. y Cuba I. (2003). Efecto de la modificación del $\mathrm{pH}$ sobre la lixiviación de cationes en columnas de suelos calizos. Estudios de la Zona no Saturada del Suelo 6, 99-104.

Huesemann M.H. y Moore K.O.J. (1993). Compositional changes during landfarming of weathered Michigan crude oil contaminated soil. J. Soil Contam. 2, 245-264. DOI: $10.1080 / 15320389309383442$

Ramírez M.E., Zapién C., Zegarra H.G., Rojas N.G. y Fernández L.C. (2009). Assessment of hydrocarbon biodegradability in clayed and weathered polluted 
soils. Int. Biodeterior. Biodeg. 63, 347-353.

DOI: 10.1016/j.ibiod.2008.11.010

Riser-Roberts E. (1998). Remediation of petroleum contaminated soils. Biological, physical and chemical processes. Lewis Publishers. CRC Press Company. Boca Raton, EUA, 503 pp.

Mihelcic J.R., Lueking D.R., Mitzell R.J. y Stapleton J.M. (1993). Bioavailability of sorbed- and separate-phase chemicals. Biodegradation 4, 141-153.

DOI: $10.1007 / \mathrm{BF} 00695116$

Montgomery D.C. (2006). Diseño y análisis de experimentos. 2da ed. Limusa, Wiley, Arizona, EUA, 686 pp.

Satyanarayana T., Bhavdish N. y Anil Prakash. (2012). Microorganisms in environmental managenent: Microbes and environment. En: Role of microorganisms in remediation of contaminated Soil (M. Das y A. Adholeya, Eds.). Springer, Nueva York, EUA pp. 113-135

Schroeder R., Rodríguez V. y Hernández L.G. (1999). Potencial de la biorremediación de suelo y agua impactados por petróleo en el trópico mexicano. Terra Latinoamericana 17, 2.

SEMARNAT (2000). Norma Oficial Mexicana NOM021-RECNAT-2000. Que establece las especificaciones de fertilidad, salinidad y clasificación de suelos. Estudios, muestreo y análisis. Secretaría de Medio Ambiente, Recursos Naturales y Pesca. Diario Oficial de la Federación. 31 de diciembre del 2002.

SEMARNAT (2003). Norma Oficial Mexicana NOM138-SEMARNAT/SSA1-2003. Límites máximos permisibles de hidrocarburos en suelos contaminados en suelos y lineamientos para muestreo y la remediación. Secretaría de Medio Ambiente, Recursos Naturales y
Pesca. Diario Oficial de la Federación. 10 de septiembre del 2013.

SEMARNAT (2008). Norma Oficial Mexicana NMX-AA145-SCFI-2008. Para la determinación de hidrocarburos fracción media por cromatografía de gases con detector de ionización de flama- Método de prueba. Secretaría de Comercio y Fomento Industrial. Diario Oficial de la Federación. 26 de marzo 2009.

Trujillo N.A., Rivera-Cruz M.C., Lagunes-Espinoza L.C., Palma-López D.J., Soto-Sánchez S. y RamírezValverde G. (2012). Efecto de la restauración de un fluvisol contaminado con petróleo crudo. Rev. Int. Cont. Ambie. 28, 4.

Walkley A. y Black I. A. (1934). An examination of the method for determining soil organic matter, and a proposed modification of the chromic acid titration method. Soil Science. An interdisciplinary approach to soil research 37, 29-38.

Wyndham R. y Costerton W. (1981). Heterotrophic potentials and hydrocarbons biodegradation potentials of sediment microorganism within the athabasca oil sands. Appl. Environ. Microbiol. 41, 783-790.

Yu K., Wong A., Yau K. y Tam N. (2005). Natural attenuation, bioestmulation and bioaugmentation on biodegradation of polyciclic aromatic hydrocarbons (PAHs) in mangrove sediments. Mar. Pollut. Bull. 51, 8-12. DOI: 10.1016/j.marpolbul.2005.06.006

Weaver R.E., Angle S., Bottomley P., Bezdiecek D., Tabatai A., Wollum., Mickelson S.H. y Bigfam J.M. (1994). Methods of soil analysis: Part 2. Microbiological and biochemical properties. Soil Science Society of America Book Series, Madison, Wisconsin, EUA, 1692 pp. 